\title{
Design of an Audio Compressor with Digital Control
}

\author{
Diseño de un Compresor de Audio con Control Digital
}

\section{Jonnathan Montenegro ${ }^{1^{*}}$}

${ }^{1}$ GRIAP-Grupo de Investigación en Acústica Aplicada, Ingeniería de Sonido, Universidad de San Buenaventura, Bogotá, Colombia.

Correspondence

Jonnathan Montenegro, San Buenaventura, Bogotá, Colombia.

Email: jmontenegro@usbbog.edu.co

Copyright : Licencia de Creative Commons Reconocimiento-NoComercial 4.0 Interna.

\section{(c) creative

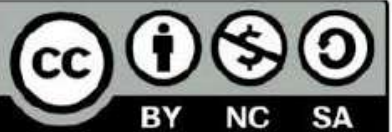

The publication of this journal is funded by Universidad ECCI, Bogotá-Colombia.

Editors: Robert Paul Salazar

Editorial assistant : Luz Adriana Suáres Suáres.

How to cite: Jonnathan Montenegro, Design of an Audio Compressor with Digital Control, TECCIENCIA, Vol. 16, No. 30, 51-64, 2021

DOI:http://dx.doi.org/10.18180/tecciencia.2021.30.4

\section{1 | INTRODUCTION}

In sound engineering, compression is a phenomenon which can be approached from the physical mechanisms of the sound particles that experiment compression and rarefaction while propagating in the acoustic mechanical wave [1]; or, a phenomenon observed in audio coding technologies related to the perceptual procedures to minimize a file volume in order to facilitate transmission and storage purposes [2], as well as its evaluation [3]; or in the HW design, a device which limits the dynamic range of an audio signal [4].

\begin{abstract}
Based on the concepts and insights of cybernetic theory and the applications of applied electronic engineering and control, this article deals with the design of an analog compressor with digital control. The main purpose of the device is to control the basic parameters of a hardware using an application on a micro-controller. The advantage of that design is the possibility of store the different analog positions into a digital position of the memory of the micro-controller, in order to call these presets whenever its needed. Further possible applications include the virtual-remote monitoring of events where the interaction between artist and sound engineering is required.
\end{abstract}

keywords: Analog compression, digital control, HW of audio, electronic micro-controllers.

\section{RESUMEN}

Este artículo trata acerca del diseño de un compresor análogo con control digital basado en los conceptos de teoría cibernética y las aplicaciones de ingeniería electrónica aplicada y control. El objetivo principal del dispositivo es controlar los parámetros básicos de un HW utilizando una aplicación en un microcontrolador. La ventaja de ese diseño es la posibilidad de almacenar las diferentes posiciones análogas en una posición digital de la memoria del microcontrolador, con el fin de realizar una llamada a estas configuraciones siempre que sean necesario. Algunas posibles aplicaciones incluyen el monitoreo virtual-remoto de eventos donde la interacción entre el artista y la ingeniería de sonido se requiere.

keywords: Compresión análoga, control digital, HW de audio, microcontroladores electrónicos.

*Equally contributing authors. 
Technologies which have been implemented throughout the years include analogue processing as well as digital schemes [5, 6, 7].This article proposes the design of an analog compressor with digital control [8]. Its main goal is to adjust the basic adjustment parameters of the device, through an application implemented on a microcontroller. The advantage is the possibility of making different types of adjustments and as well to save the analog positions of the controls in a memory with the possibility to call them afterwards when necessary [9].

The dynamic audio signal compressor is a primary system in audio production processes [10]; it is an essential element in the electroacoustic chain that transmits the signal, it attenuates the dynamic range of the signal, understood as the logarithmic relationship in decibels between the maximum and minimum signal level, preventing distortions or damage to the equipment that can be caused by saturations or damage. These phenomena are sometimes called "spikes" [11].

Currently, compressors can be analogous, whose structure is made up of electronic elements, in other words, hardware. There are also digital ones which act through an algorithm. The main objective of the present work is to design a compressor, with the main parameters (such as threshold, ratio, and output gain) in each band controllable through MatLab programmed user interface [12]. This constant interface of buttons indicated to each compression parameter, step indicators, so that the user interacts and processes the audio of his particular taste, in an intuitive manner. It should be noted that the digital control mentioned above does not affect the audio signal, it only acts on the specific compression parameters.

The proposed digital control provides the following advantages: (i) it avoids the need to manually adjust analog potentiometers, (ii) allows to save compression settings to a list to be easily and quickly activated on the HW (analog circuit) with a single touch within the user interface. The implementation of the digitally compressor consists of two main development stages. The processes are executed independently. First, there is the design of the analog compression circuit, that is the set of electronic components that process dynamically the audio signal. Secondly, a digital control system is implemented that must be compatible with the analog circuit by means of integrated circuits and the user interface. In summary, the digital control system is capable of activating the analog compression circuit. Among the most relevant applications of dynamic range compression devices, hearing aids are currently the most prominent ones [13, 14], as well as Speech Processing [15].

\section{2 | CIRCUIT DESIGN}

\subsection{HW implementation}

The analog state of the compressor consists of three main control parameters (threshold, ratio and output gain); for each band. These are controlled through a user interface programmed in MatLab. The interface consists of buttons for each compression parameter, step indicators, so that the user can act and process the audio as the like in an intuitive manner.

\subsection{1 | Design of the circuit of dynamic processing of the signal (compressor)}

It was decided to use a feedforward topology for the design of this stage, and for the amplification stage, due to its versatility, a VCA (Voltage Controlled Amplifier) will be implemented, after having analyzed the other options, among which FET, optical, VariMu and digital types are at disposal. Fig. 3 shows the schematic for the analog circuit of the compressor. The integrated circuit for the development of this stage is the THAT 4301 (see Fig. 1), which has within its structure a VCA circuit, an RMS signal detector and three operational amplifiers. 


\subsection{Description of the performance of the stages of $\mathrm{Cl}$ THAT 4301}

\subsection{1 | VCA Circuit}

The VCA circuit of the 4301 chip is based on the log-antilog topology used in THAT 2180 series integrated circuits. This uses a fully complementary topology double FET configuration. An important parameter is the input to the VCA device. The signal entering it through pin 17 (VCA IN) is the current. This input point is a virtual ground, so for its normal operation an input resistance R1 must be placed in Fig. 3. To avoid DC shifts, since any of these shifts will be modulated in later stages, this input must be coupled with a capacitor. The VCA output is current with reverse polarity to that found at the input of the circuit. For this reason, it becomes necessary to convert this signal into a voltage. Internally, the VCA output is connected to the OA3 operational amplifier, and a resistance (feedback resistor) must be connected between its output and the VCA output, used to determine the conversion ratio or gain of the direct loop. The VCA gain is controlled by the voltage applied to Ec-, Ec+, and the SYM pin. The gain in $\mathrm{dB}$ is proportional to the difference between (Ec +) - (Ec-). The proportionality constant is $-6.5 \mathrm{mV} / \mathrm{dB}$ for the voltage in Ec- and $6.5 \mathrm{mV} / \mathrm{dB}$ for the voltage in Ec+ and the pin SYM.

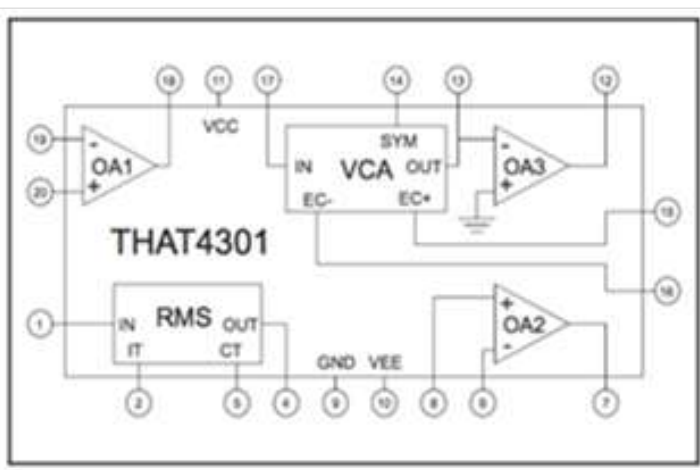

FIG. 1 Block diagram of CI THAT 4301.

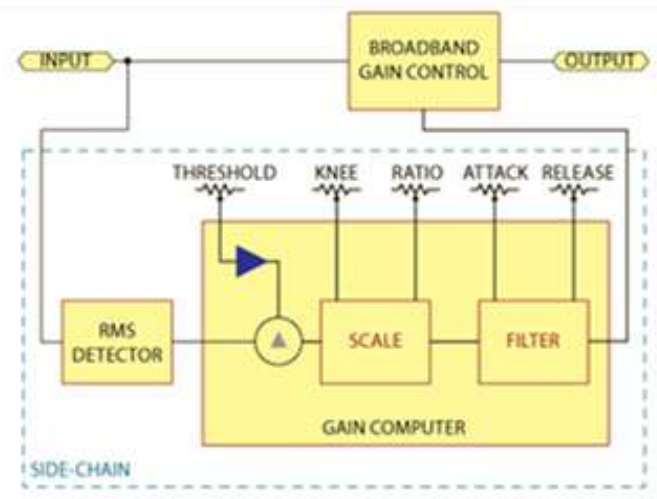

FIG. 2 Block diagram of the compressor.

\subsection{2 | RMS Detector}

The 4301 integrated circuit RMS detector computes the RMS level by rectifying the input current signals converting the rectified current to a logarithmic voltage and applying that voltage to a filter in the logarithmic domain [16]. The output signal is a DC voltage proportional to the $\mathrm{dB}$ level of the RMS value of the current input signal. The AC signal is attenuated by a filter with a single-pole, with a cutoff frequency that is adjusted by an external capacitor and DC current. The design procedure is the following, with the schematic circuit for the design as shown in Fig. 3.

We set $V C C=15 \mathrm{~V} ; \mathrm{VEE}=-\mathrm{VCC}, \mathrm{VD}=0.7 \mathrm{~V} V t=26 \mathrm{mV}$ and Vinre $f=0.5 \mathrm{~V}$ and $\mathrm{rms}=-6 \mathrm{dBV}$. The voltage constant of voltage control of the 4301 is $6.5 \mathrm{mV} / \mathrm{dB}=$ KGain. IT $=7.5 u A$ according to the data sheet recommendation, $R 9=(V E E+2 V D) / I T=2.18 M W \approx 2.18 M \Omega$.

The detector release time should now be found. A value of $120 \mathrm{~dB}$ per second is assumed by the manufacturer to be the best decision without the introduction of noticeable distortion. RelRate $=120 \mathrm{~dB} / \mathrm{s}$ is then defined. The voltage at the detector output changes with a constant defined as: Vc=Kgain * RelRate = $6.5 \mathrm{mV} / \mathrm{dBx} 120 \mathrm{~dB} / \mathrm{s} .=780 \mathrm{mV} / \mathrm{s}$. The $C 6$ capacitor, which fixes the release parameter is $C 6=I T / V c=$ $9.61 u F \approx 10 u F$.

The time constant of the RMS detector is given by the equation $0.026 C T / I T=34.66 \mathrm{~ms}$. A $20 \mathrm{k} \Omega$ value for the input resistance $R 1$ is assumed since this value is ideal for input voltages up to $10 \mathrm{VRMS}$, and it is needed to convert the input AC voltage into a current within the linearity range of 4301 (it is recommended to keep 
the values of the currents below $1 \mathrm{~mA}$ in the input of the VCA, to improve the performance in relation to the distortion. The $\mathrm{C} 1$ input (coupling) capacitor is used to block the DC signal from previous stages. $C 1$ together with $R 1$ set the limit cutoff frequency of the circuit at low frequency. $V F 1$ is voltage on input signal, and $V F 2$ is voltage at the output signal. The frequency is $F c=1 /(2 \pi R 1 C 1)=0.16 \mathrm{~Hz}$. The VCA output is directly connected to the $\mathrm{U} 2$ amplifier configured as an inverter current-to-voltage converter. Components $R 4$ and $C 3$ set the time constant for this conversion. The simplest way to set these components is to recognize that when the VCA gain has a unity gain, the gain ratio is given by $R 4 / R 1$. Capacitor $C 3$ is required for the stability of the feedback circuit. The VCA output has approximately $45 \mathrm{pF}$ capacitance to ground, a value which is neutralized with a $47 p F$ capacitor $(C 3)$ in parallel with $R 4$.

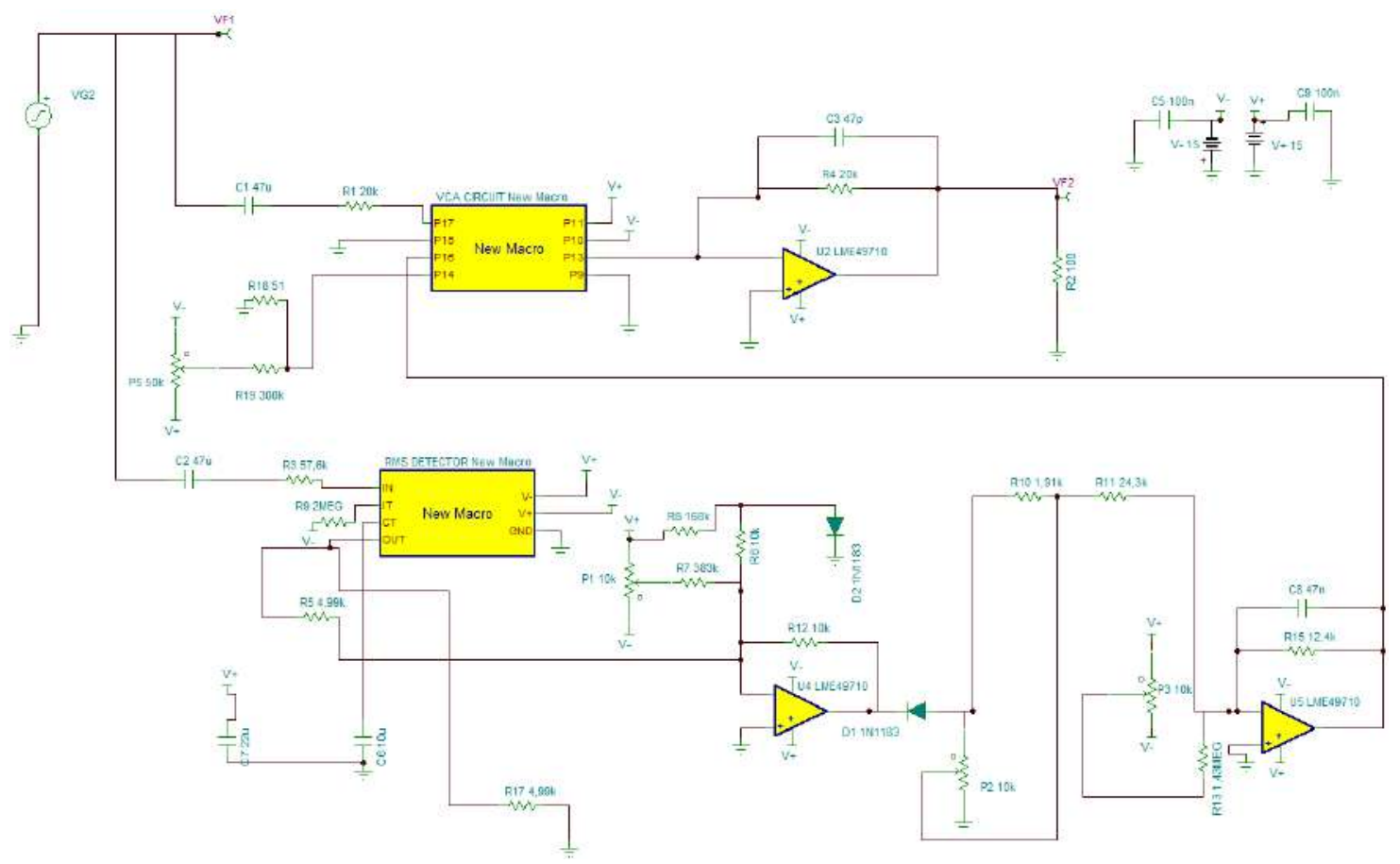

FIG. 3 Schematic diagram of the compressor with feedforward topology for each band.

\subsection{3 | Control path}

The compressor design requires the compressor to reduce its gain if the signal exceeds the threshold set. The output of the RMS detector represents the level of the input signal at various levels, but compression only occurs when the level exceeds the threshold. Amplifier U4 is configured as a variable threshold detector that locks information from low level signals and passes only information from signals that exceed the threshold. This amplifier has a negative gain of -2 given by the relationship between $R 12(10 K \Omega)$ and R5 (4.99K $\Omega$ ). The positive signals that feed the U4 come out negatively, due to the inverting configuration of the amplifier, and cause D2 to be polarized in direct and the output to be -2 times the output of the RMS detector. The negative signals coming out of the detector positively feed $\mathrm{U} 4$ and cause $0 \mathrm{~V}$ to come out of the amplifier $\mathrm{U} 4$ and no signal goes to the detector threshold output.

To vary the threshold, a potentiometer $P 1(10 k \Omega)$ is required, to which a resistance $R 7(383 k \Omega)$ is placed, connected to the central tab, which allows the current to change by $\pm 39.2 u A$ to the inverter input of the $\mathrm{U} 4$, and the same amount of current is required, but in reverse polarity for a balance to be made. If a $4.99 \mathrm{k} \Omega$ resistor is placed, the required voltage at $\mathrm{R} 5$ is $\pm 195 \mathrm{mV}$, which represents a variation of $\pm 30 \mathrm{~dB}$ of change in the input level of the RMS detector.

The detector threshold output represents the signal level above a given threshold, with a constant of 13 
$\mathrm{mV} / \mathrm{dB}$ of the ratio of $[R 12 / R 5] x(6.5 \mathrm{mV} / \mathrm{dB})$. The signal then goes to the compression ratio control (potentiometer $P 2$ ), which variably attenuates the signal going to the amplifier $U 5$. The gain of this amplifier seen from the central pin of $P 2$ is $R 15(0.5)$, precisely the inverse gain of the U4 amplifier. In this way, the compression control allows the user to vary the gain above the threshold, in values between the output of the RMS detector and the output of the U4 amplifier, from 0 to a maximum of 1 .

The VCA gain control constant which is $6.5 \mathrm{mV} / \mathrm{dB}$ is equal to the scaling constant of the RMS detector. Therefore, at maximum compression above the threshold, each increase in the input signal exerts a $6.5 \mathrm{mV}$ increase in the output of the $\mathrm{U} 5$ amplifier, which in turn causes a $1 \mathrm{~dB}$ attenuation in the VCA gain. In accordance with this arrangement, the output does not increase abruptly despite large variation in the input level above the threshold. This may be called infinite compression or limitation. On the other hand, the intermediate positions in the compression or (ratio) control, a $1 \mathrm{~dB}$ increase in the input signal will cause less than $1 \mathrm{~dB}$ of gain attenuation when varying this parameter.

The gain control which is operated with potentiometer $P 3$ is used to give the circuit a static gain or attenuation in the signal control path. This control adds up to $\pm 130 \mathrm{mV}$ offset to the output of the U5 amplifier, equivalent to $\pm 20 \mathrm{~dB}$ change in VCA gain (this value comes from dividing $130 \mathrm{mV} /(6.5 \mathrm{mV} / \mathrm{dB})$ ). $C 8$ must be used in the circuit to attenuate the noise of the op amps $U 5$ and $U 4$, as well as the resistors used in the signal side-chain.

With these adjustments in the design, the compressor controls it as follows: Threshold, which can be varied between $\pm 30 d B$, ratio (or compression ratio) that can be varied from 1: 1 (where there is no compression), to a range of $\infty: 1$ (equivalent to a limitation) and gain (static gain) which can add up to $\pm 20 \mathrm{~dB}$.

\section{3 | SIMULATION OF COMPRESSION CIRCUITS}

It was decided to use the TINA-TI free software to run the simulations of the specific compression circuits, since it is possible to visualize the responses of the specific threshold, ratio and gain parameters. Simulation of the transient response of the circuit is observed in Figs. 4 and 5.

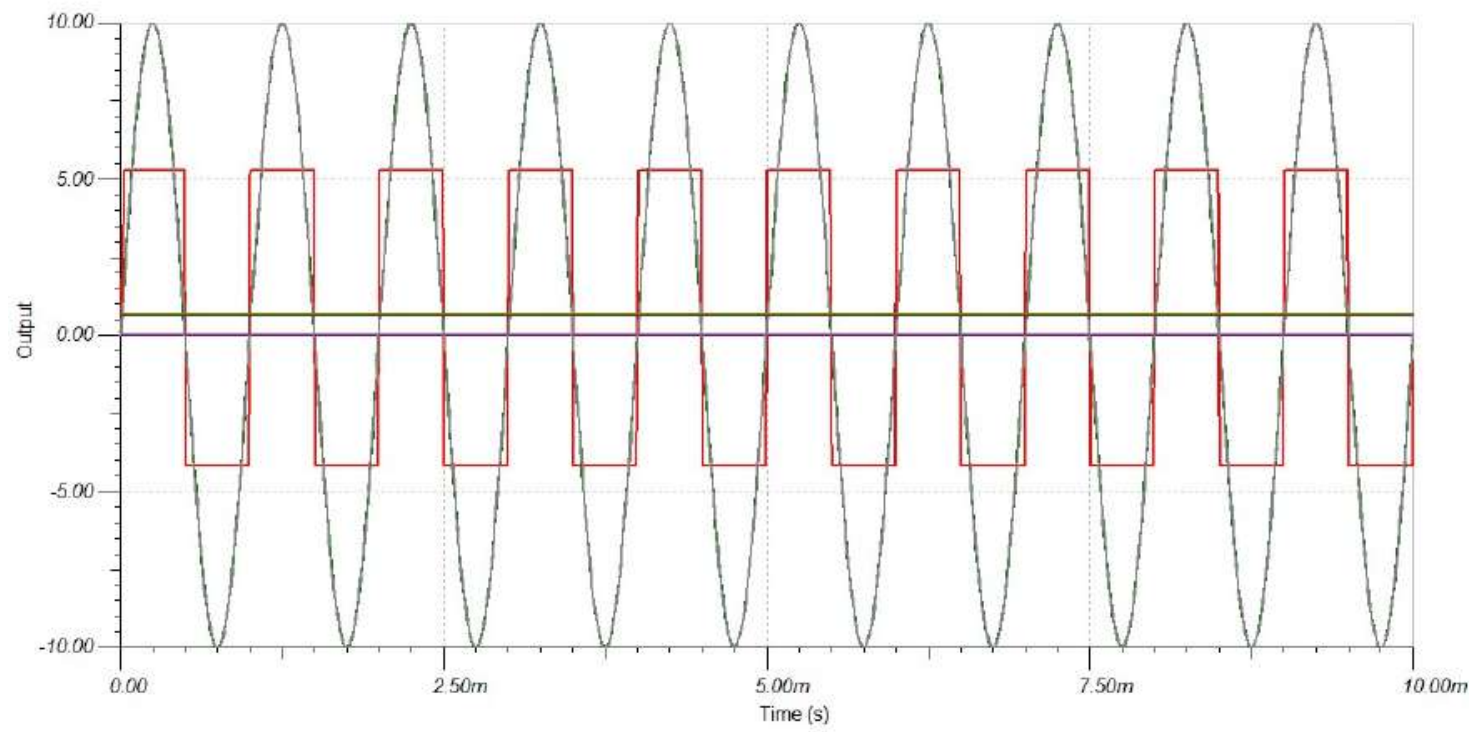

FIG. 4 Simulation of Compressor/Limiter with $\infty: 1$ ratio.

Where VF1 is the voltage generated at the source and VF2 is the measured voltage at the output of the compressor. 


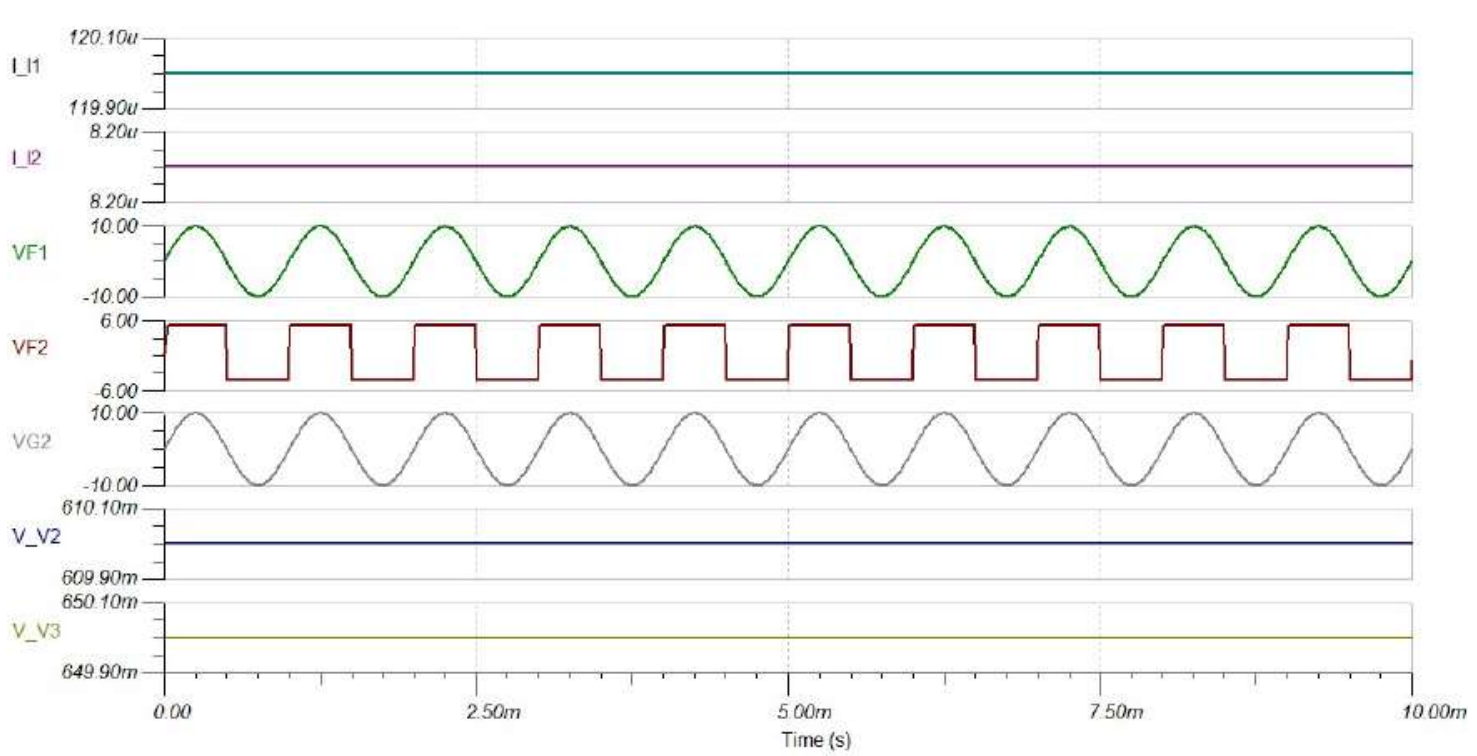

FIG. 5 Simulation of compressor/limiter with $\infty: 1$ ratio, separated signals.

\section{4 | MEASUREMENT OF PERFORMANCE PARAMETERS OF THE ANALOG PROTOTYPE}

After performance verification through the simulation of the compressor base circuit, the implementation of the circuit is done on the breadboard, and the comparison between the performance of the prototype against the required design parameters is performed. A test signal is generated for the measurement of the different parameters in a digital audio workstation (DAW), in this case ProTools was used. This signal is a $1 \mathrm{kHz}$ tone with a -20 dBFS value. Analog output 5 of the RME Fireface 400 interface is used which generates an RMS voltage of $273 \mathrm{mV}$ or $-9 \mathrm{dBu}$ (see Fig. 6).

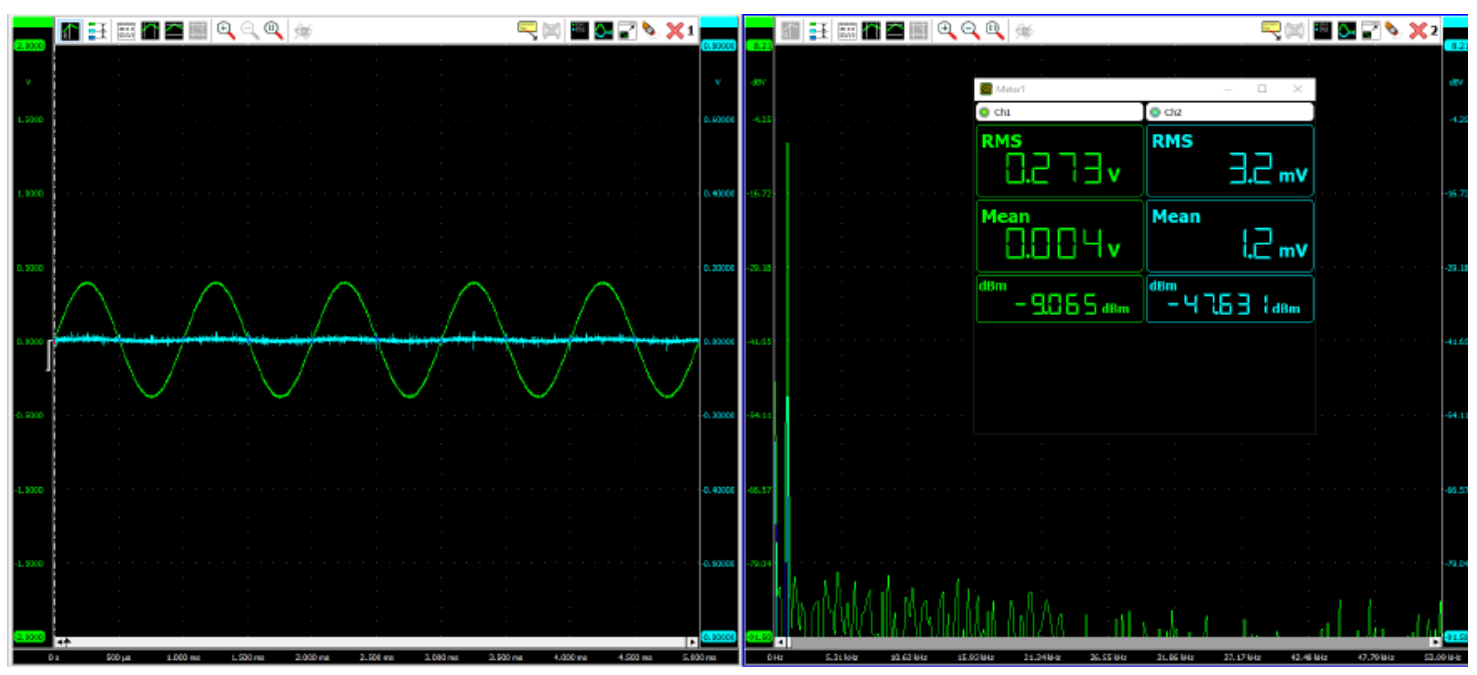

FIG. 6 Simulation of compressor/limiter with $\infty: 1$ ratio, separated signals.

\subsection{Attack and Release measurement}

To measure attack and released parameters, the test signal must be modified so that the circuit can detect changes in input levels It is decided to use the test signal with a duration of 20 s (see Fig. 7), and that every $5 \mathrm{~s}$ 
change, the level from -20 dBFS a -14 dBFS changes (an increase of $6 \mathrm{~dB}$ ).

TAB LE 1 Measurement signals for the attack/release times with 5 different positions in controls.

\begin{tabular}{|l|c|c|c|}
\hline Signal & THRESHOLD & RATIO & GAIN \\
\hline 1 & Maximum (no compression) & $1: 1$ & OdB \\
\hline 2 & Medium & $4: 1$ & OdB \\
\hline 3 & Medium & $\infty: 1$ & OdB \\
\hline 4 & Minimum & $4: 1$ & OdB \\
\hline 5 & Minimum & $\infty: 1$ & OdB \\
\hline
\end{tabular}

FIG. 7 Measurement signal for the attack/release times visualized in the DAW ProTools.

Five positions are stablished on the control potentiometers to determine how these settings modify the times. As seen in Fig. 8, the times are very similar, regardless of the position of the threshold, ratio and the gain controls. The configurations in the controls are in Table 1.

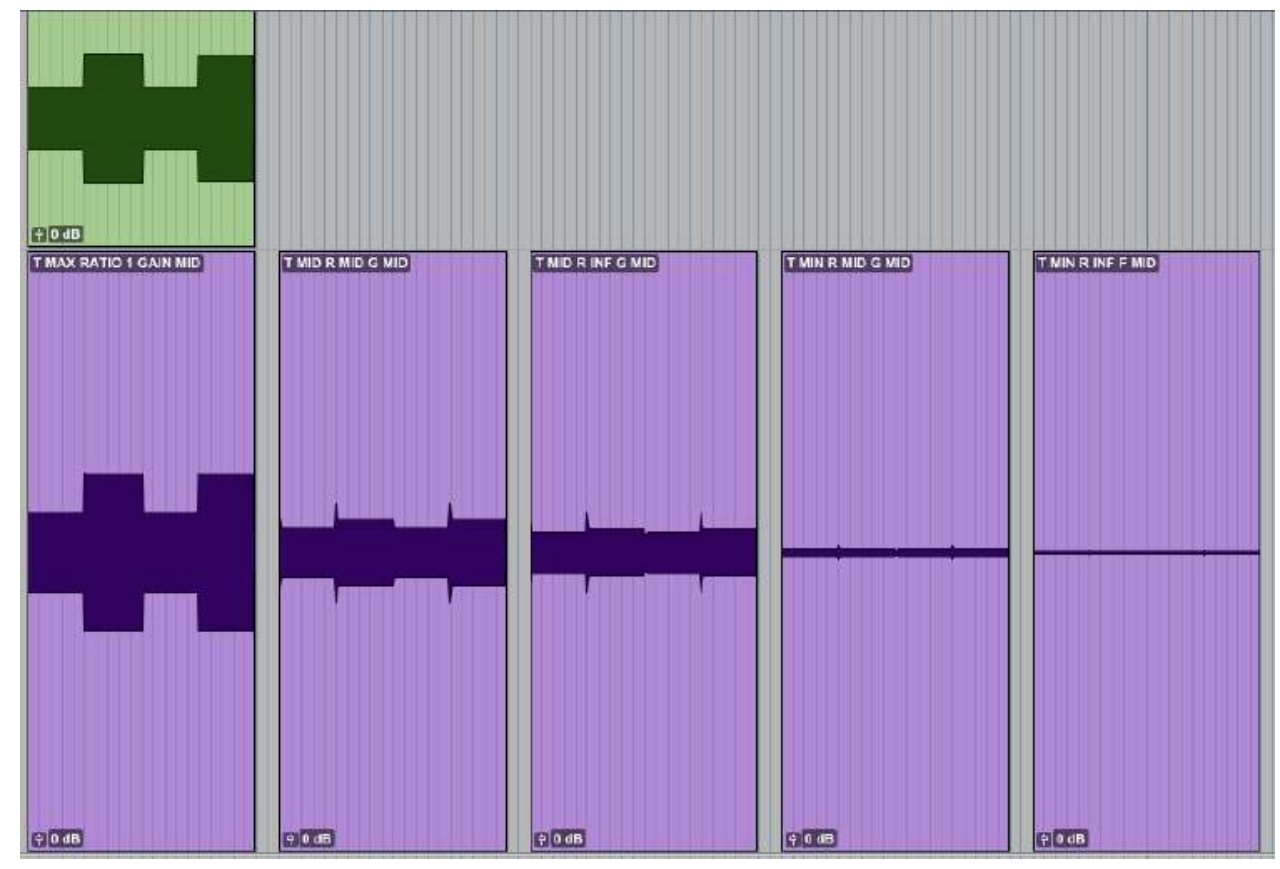

FIG. 8 Measurement signals for attack/release times with 5 different positions visualized in DAW.

To determine these times with better precision, the time in which the device starts working after having exceeded the threshold, and the time at which the prototype stops working and returns to the original signal, is measured within the DAW. According to the graphs, this time is close to $300 \mathrm{~ms}$, if the times of these 3 signals are averaged, it would be $273.6 \mathrm{~ms}$. For the release time, it is preceded in the same manner, analyzing in the DAW and measuring the recovery times of the circuit. 


\subsection{Control measurement of compression relations (ratio)}

To finish this parameter, a measurement of the levels is made in $\mathrm{dBu}$ of the input against the output of the device, by means of a digital oscilloscope, with the previously used test signal, which changes its gain value every 5s. The compressor controls are located in two different positions to output 2 ratio values for the Mid positions (equivalent to $4: 1$ according to design) and the $\max$ (equivalent to $\infty: 1$ ) respectively, and these values are averaged to give an estimate of the device's operation. Signals 2 and 4 in Table 1 are used to make these measurements for a ratio of $4: 1$ and Signal 5 for a ratio of $\infty: 1$.

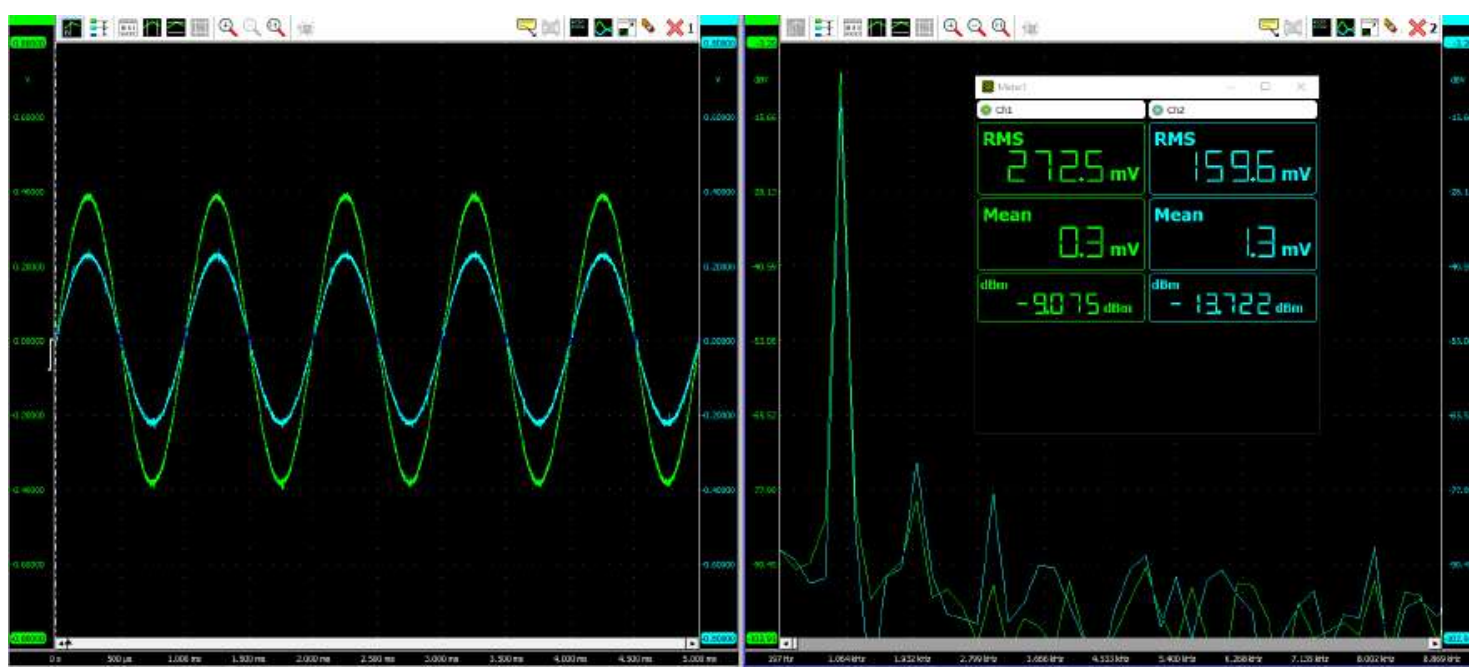

FIG. 9 Measurement signal 2 with - 20 dBFS value.

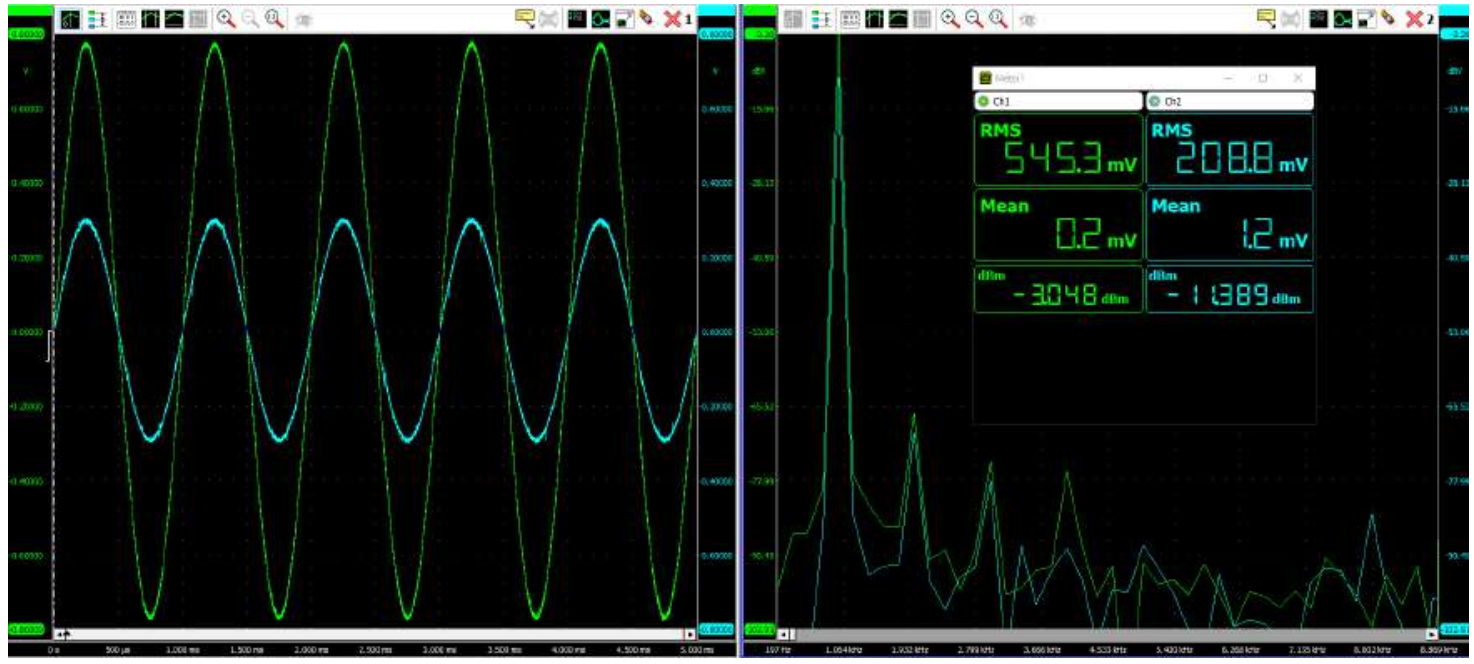

FIG. 10 Measurement signal 2 with $-14 \mathrm{dBFS}$ value.

In order to determine the ratio value, the dBu change in the input signal (green colour), with respect to the output signal (in blue colour) for the Figs. 9 and 10. Therefore,

- The change in $\mathrm{dBu}$ of the input signal $=(9.075-3.048)=6.027$

- The change in $\mathrm{dBu}$ of the output signal $=(13.72-11.38)=2.34$

- Ratio $\mathrm{dB}=\mathrm{dB}$ in $/ \mathrm{dB}$ out $=2.57$ 
For the signal No. 4, same procedure is applied and the results are:

- The change in $\mathrm{dBu}$ of the input signal $=(9.075-3.049)=6.026$

- The change in $\mathrm{dBu}$ of the output signal $=(30.62-29.37)=1.25$

- Ratio $\mathrm{dB}=\mathrm{dB}$ in $/ \mathrm{dB}$ out $=4.82$

By averaging these two ratios, you have a value for the Mid position of the prototype ratio control of 3.69:1, a value very close to that of design which is $4: 1$. A similar procedure is performed to measure the ratio control at its maximum value, which in theory yields a $\infty: 1$, which is equivalent to the device behaving as a limiter.

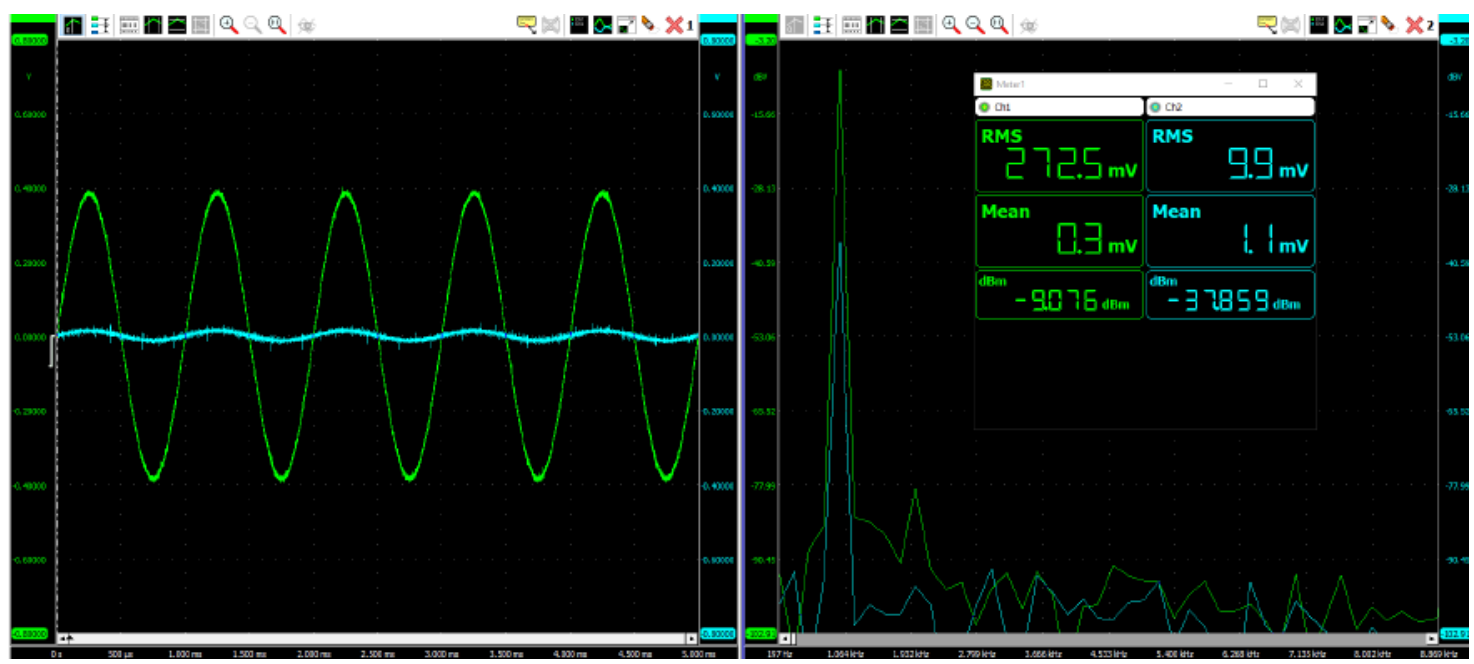

FIG. 11 Measurement signal 2 with - 20 dBFS value.

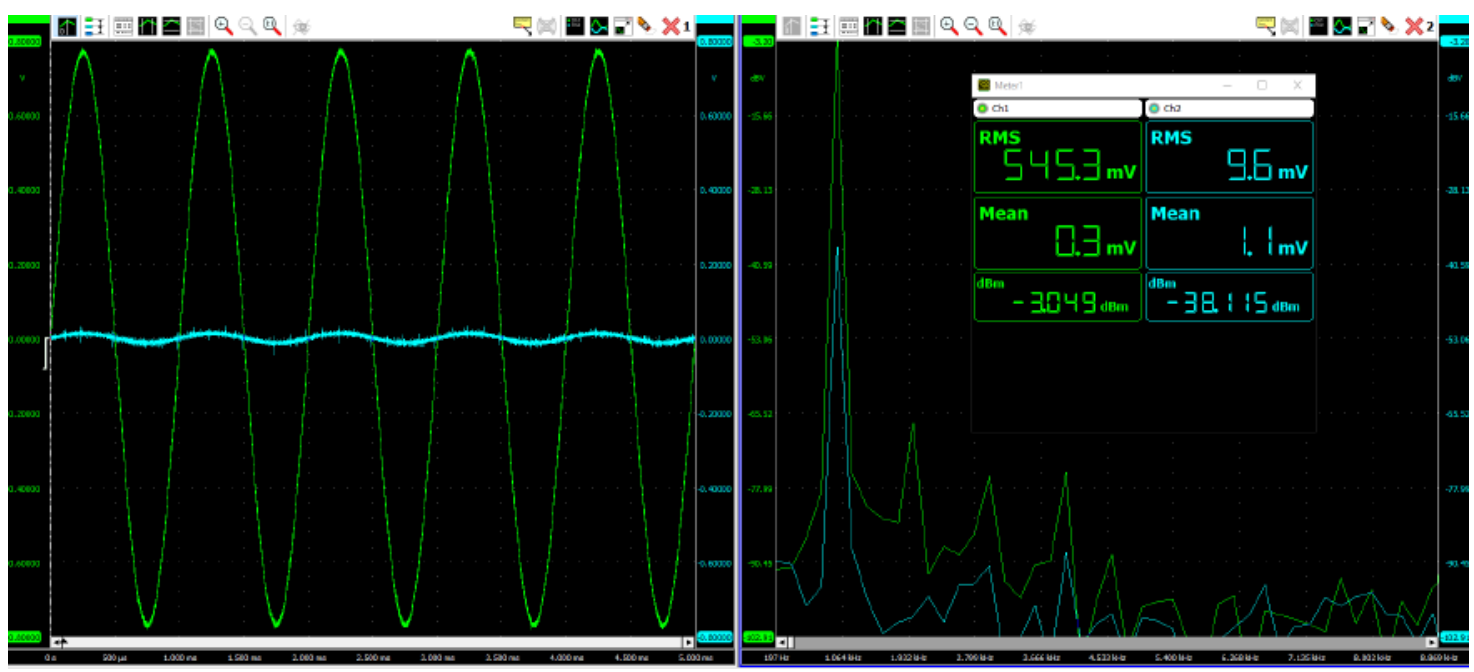

FIG. 12 Measurement signal 2 with -14 dBFS value.

From signal 5 (see Fig. 11 and 12), the values are obtained:

- Change in dBu of the input signal $=(9.076-3.049)=6.027$

- Change in dBu of the output signal $=37.85-38.11)=0.26$

- Ratio $\mathrm{dB}=\mathrm{dB}$ in $/ \mathrm{dB}$ out $=23.18$ 
For the maximum position, the value approaches to the design. Due to the fact that it is established a relation of more than 10:1 in a compressor, then it behaves as a limiter.

\section{5 | DIGITAL CONTROL SYSTEM}

Two microcontrollers are tested, namely the Arduino Mega 7627 board and Tennsy 3.2, both work correctly; however, it was decided to use Arduino to have the libraries with the MatLab platform which is where the user interface is designed. Thus, MatLab, with its library that allow compatibility with the Arduino Mega system, manages to establish communication with the analog circuit of the compressor. It should be clarified that the proposed digital system depends on a wired connection with the analog section of the compressor, in a later phase, the project will be complemented with a wireless system. The following block diagram exposed in Fig. 13, outlines the digital control system.

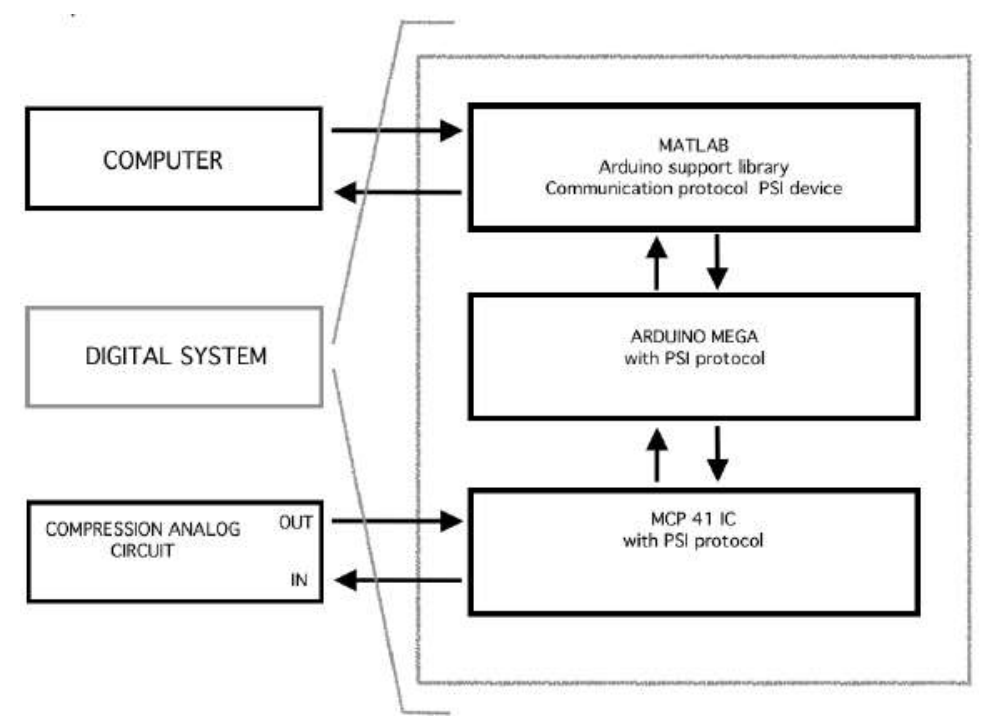

FIG. 13 System block diagram of the proposed compression system.

Additionally, in the proposed design of the compressor, two ways are established for signal control. At first, analog potentiometers in the conventional way, as well as the digital control of the parameters through the MatLab graphical user interface and the MCP 41 integrated circuits, which together allow changing resistivity to define new compression settings. This digital system facilitates the storage of these configurations to be used later when required.

\section{1 | MatLab to Arduino code}

As it shown in the Fig. 13, the design interface user in MatLab is connected with the Arduino system, which at the same time is connected to the analog circuit of the compressor. These ensembles of interconnected systems set the analog compressor with digital control and present the following main functions: Digital communication with hardware through the integrated circuits MCP 41 which vary their resistivity with the established PSI protocol. This protocol enables that the integrated circuit holds its last position.

- Allows compression with digital control saving parameters set by the user for later use.

- Accurately access different compression options by moving digital faders defined by steps according to the MCP 41 integrated circuit with PSI protocol.

- Using a menu, the user can load previously saved compression settings; if not, he can start creating his own "preset". 
Fig. 14 shows the opening code of the Arduino Mega2560 object whose function is to digitally control the compressor. The variable d_pot to $d_{-}$pot8 represent the 9 MCP 41 integrated circuits with the SPI protocol.

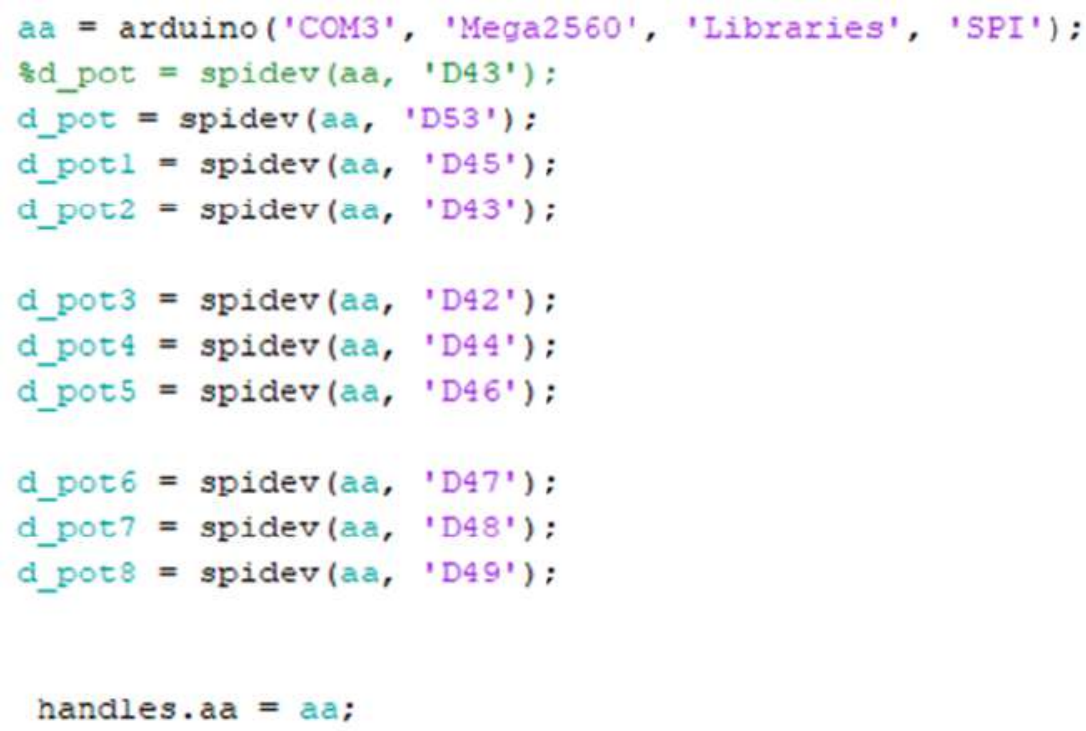

FIG. 14 Arduino Mega opening code, libraries and SPI protocol.

\section{I GRAPHIC USER INTERFACE FOR THE DIGITAL CONTROL OF THE COMPRESSOR}

The importance of a digital control for precision purposes is emphasized, in addition to offering the possibility of saving predetermined compressions. An intuitive interface with the main compression, threshold, ratio and output gain parameters is presented, making it a GUI similar to the typical front panel of an analog compressor. The proposed design is the one presented in Fig. 15.
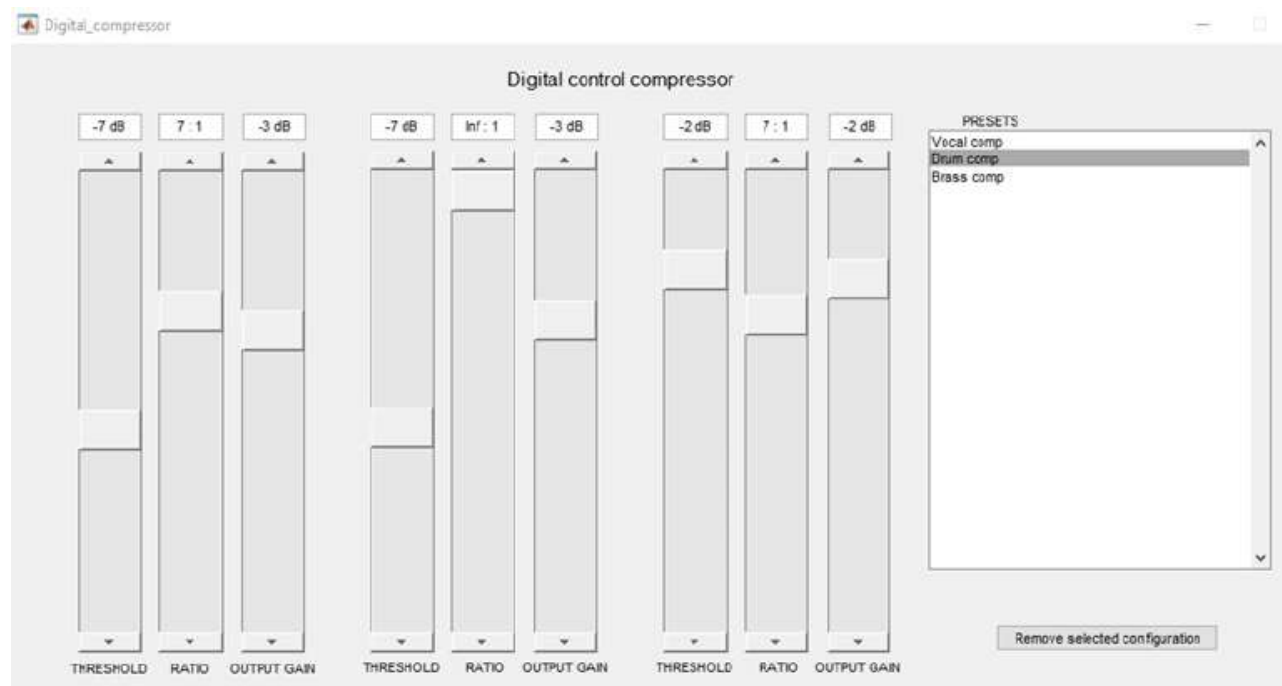

SAVE

Save configuration

Losd configuration

FIG. 15 Graphic unit interface (GUI) in MatLab. 
The implicit GUI programming in MatLab used the libraries that Mathworks provides for compatibility with the Arduino Mega system. These libraries are those that allow you to control the functions of the Arduino board from the MatLab code. For this connection, the USB cable provided by the Arduino is necessary, and for the future work, for the optimization of this project, it is planned to turn it into a completely wireless system.

\section{Conclusions}

The proposed digital control provides the following utilities: it avoids the need to manually adjust analog potentiometers, obviating their manipulation. Additionally, it allows you to save compression settings in a list to be easily and quickly activated in the hardware (analog circuit) with a single touch within the user interface.

The use of VCA integrated circuits and RMS value detectors allow the design of devices for the dynamic control of a signal, either in feedback or feedforward topologies. When assembling the two stages, analog and digital, there are some drawbacks in the handling of voltages, since some integrated circuits as the MCP41010, MCP410100 and MCP41050 have low tolerance for voltage differences; for this reason it was decided to use the high-voltage integrated one, namely MCP41010HV, MCP410100HV, MCP41050HV. This allows the system to be strengthened to be used reliably.

\section{References}

[1] P. Mittal, Oscillations, Waves and Acoustics. New Delhi, India: Int. Publ. House.

[2] K. Brandenburg, "Mp3 and aac explained," in 17th Int, Conference: High-Quality Audio Coding.

[3] M. Martínez, "Evaluation of audio compression artifacts," Acta Polytechnica, vol. 47, no. 1. DOI: 10.14311/ 906

[4] D. Wang, Y. Wei, Y. Wang, and J. Wang, "An audio distortion dynamic range index for evaluating the performance of audio systems," Applied Sciences. DOI: 10.3390/app10228022

[5] D. Giannoulis, M. Massberg, and J. Reiss, "Digital dynamic range compressor design - a tutorial and analysis," Journal of the Audio Eng. Society. https ://www . aes . org/e-lib/online/browse. cfm?elib=16354.

[6] R. Lyons, Understanding Digital Signal Processing. Prentice Hall.

[7] A. Oppenheim and R. Schafer, Digital Signal Processing. Prentice Hall.

[8] J. Kates, "Principles of digital dynamic-range compression," Trends in Amplification. DOI: 10.1177/ 108471380500900202

[9] B. Blesser and K. Baeder, "A new approach to dynamic range compression for audiosystems," in 35th AES Convention. https://www. aes.org/e-lib/online/browse.cfm?elib=1410.

[10] M. Massberg, Investigation in Dynamic Range Compression. MSc Thesis. London: Queen Mary University of London.

[11] G. Davis and R. Jones, "Sound reinforcement handbook."

[12] U. Zolzer, Digital Audio Effects. New York: John Wiley and Sons.

[13] K. Chung, "Wind noise in hearing aids: I. effect of wide dynamic range compression and modulation-based noise reduction," Int. Journal of Audiology. DOI: 10.3109/14992027.2011.609181

[14] J. Kates, "Understanding compression: modeling the effects of dynamic-range compression in hearing aids," Int. J. Audiol, pp. 395-409,. DOI: 10.3109/14992020903426256 
[15] P. Souza, "Effects of compression on speech acoustics, intelligibility, and sound quality," Trends Amplif, pp. 131-165,. DOI: 10.1177/108471380200600402

[16] J. Abel and D. Berners, "On peak-detection and rms feedback and feedforward compression," in 115th of the AES Convention.

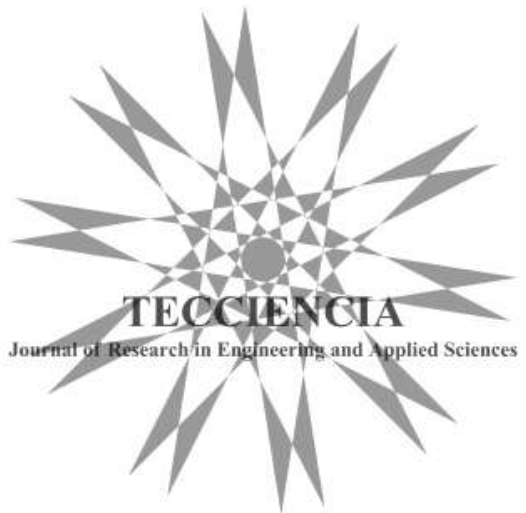




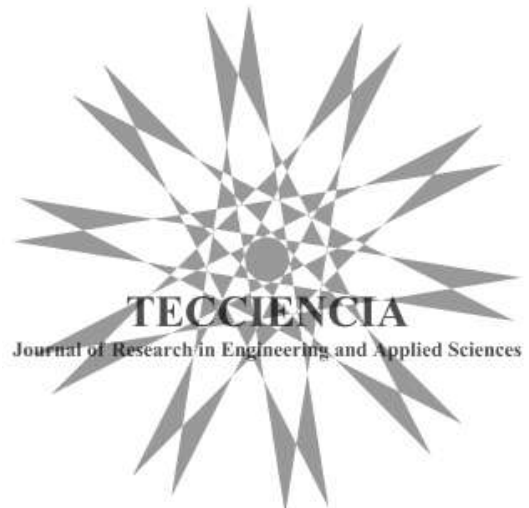

